\title{
Variously Worldwide Types of Deregulated Electricity Markets and Their Respective Transmission Congestion Management Schemes
}

\author{
Jiawei Zhao \\ University of Strathclyde \\ Glasgow, United Kingdom \\ jiawei.zhao.2013@uni.starth.ac.uk
}

\author{
Kwok Lun Lo \\ University of Strathclyde \\ Glasgow, United Kingdom \\ k.lo@strath.ac.uk
}

\author{
Jianfeng Lu \\ University of Strathclyde \\ Glasgow, United Kingdom \\ jianfeng.lu@strath.ac.uk
}

\begin{abstract}
In this paper, four practical and successful deregulated electricity markets: the old England \& Wales Pool, the British Electricity Trading and Transmission Arrangements (BETTA), Nordic Pool and PJM interconnection will be briefly explained and discussed on their histories, structures, rules and also advantages and disadvantages. The old England \& Wales Pool was a typical Pool type market which was one of the first successful electricity market deregulations but now had been replaced by the BETTA which is based on the bilateral and balancing mechanism. Nordic Pool and PJM are hybrid markets which combine both pool and bilateral principles. With maturing deregulated mechanism, transmission congestion becomes an urgent problem for all markets. Uniform marginal price, locational marginal price and zonal price are three typical methods in Pool and Hybrid market for congestion management. Locational marginal price is regarded as more efficient than other two when it comes to the market power dilution. In bilateral market, four transaction curtailments based on four rules: first come first serve, pro rata, minimum-net curtailment and price based are utilized to relieve congestion.
\end{abstract}

Index Terms-- Deregulated Electricity Market, Transmission Congestion Management, Uniform Marginal Price, Locational Marginal Price, Zonal Price and transaction curtailment.

\section{INTRODUCTION}

Since 1980s, many countries started to unbundle their vertically integrated power utility to a competitive market environment because of the new requirements for energy industry such as reducing the cost, improving efficiency and maintaining the long-term development [1]. People believe that with the introduction of competition, liberalized electricity market will theoretically enable to satisfy the above requirements thus electricity price could get close to the marginal cost of generation, companies will minimize their production cost and customers could get a cheaper price for electricity [2]. The objective of energy industry restructure is to create a competitive environment for electricity trading in order to improve social welfare [3].

In the deregulated electricity market, transmission congestion is the major issue which could causes power system crisis. In old power structure, the system operator (SO) only needs to re-dispatch the generation schedule until the congestion has been released. However, with the deregulation process, congestion management becomes more complex since transmission network has to be opened to all market participants [4].
This paper introduces four typical worldwide electricity markets and their respectively transmission congestion management schemes.

\section{GlobAl EXPERIENCE OF COMPETITIVE ELECTRICITY MARKETS}

A. UK

- The old England \& Wales Pool

On $31^{\text {st }}$ March 1990, electricity industry in England and Wales started to be restructured with new mechanism [5]. After deregulation, the dominated vertically integrated industry Central Electricity Generating Board (CEGB) in England and Wales had been split into three generating companies: National Power, Powergen and Nuclear Electric [5]; one transmission company: the National Grid Company and 12 regional electricity companies (RECs) which were regarded as local distribution systems [1]. An electricity pool market had been established which arranged and managed electricity trades in England and Wales with wholesale market mechanism. Figure 1 shows the structure of electricity industry at privatization [5].

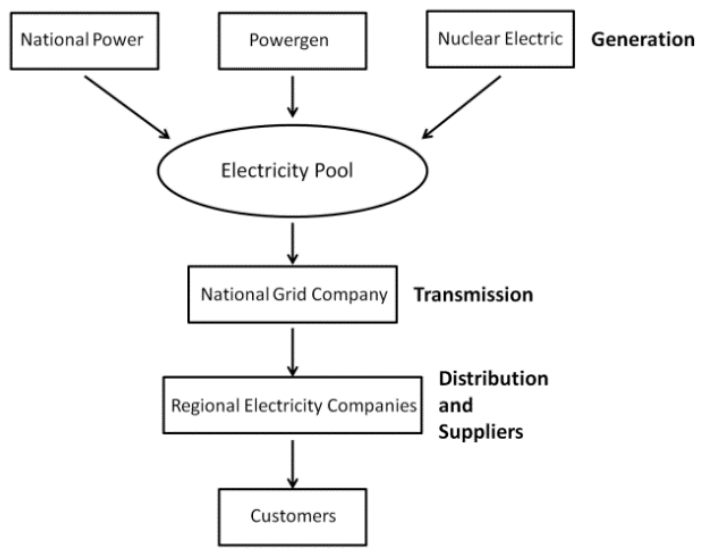

Fig. 1. The Structure of Electricity Industry in England \& Wales.

The old England \& Wales Pool is a typical mandatory pool market which means every energy trade has to be transacted only inside the pool market [2]. By 10 a.m. each day, generation companies should send generation output quantity for the following day and bid price of electricity provided on the following day to the $\mathrm{SO}$, here is the National Grid Company, who already forecasted the power demand quantity 
in each half hour period [1]. Then the SO will start to accept bids from the cheapest price to higher price until the forecasted demand is satisfied. Then, the SO will sort out a bids list which contains names of the generation companies who have been chosen to generate electricity on the following day. Those generation companies were called "in merit" generation companies which means their bids have been accepted by the SO and those who have not been accepted by the SO were called "out of merit" generation companies [2]. At last, the SO will set prices for all pool market participants. System Marginal Price (SMP) is the price which bidden by the most expensive generation company in the bids list [1]. In the pool market, the SO will pay for the Pool Purchase Price (PPP) to all the in merit generation companies. The Pool Purchase Price (PPP) is the System Marginal Price (SMP) plus Capacity Payment (CP) which is defined with Loss of Load Probability (LOLP) and Value of Loss Load (VOLL) [1]. On the other side, suppliers will pay for the price called Pool Selling Price (PSP) to purchase the electricity power from the pool [1]. The PSP is the PPP plus the Uplifts. The following equations defined the relationships between those prices [6]:

$$
\begin{gathered}
P P P=S M P+C P \\
C P=L O L P \times \max (0, V O L L-S M P) \\
P S P=P P P+\text { Uplifts }
\end{gathered}
$$

$\mathrm{CP}$ is an incentive which is used to reward the generation companies who declare that their capacity is available regardless of whether they are required to or not. LOLP is the probability that electricity power capacity is unable to support the actual demand. LOLP is predicted in each half hour.

The pool structure is regarded as an innovation for energy industry deregulation [5]. However, electricity price decrease is slower than generator cost reduction and customers are unable to access to the deregulation benefits. The biggest reason is that three dominant generation companies exercise their market power and determine price most of the time [7].

\section{- The British Electricity Trading and Transmission Arrangements (BETTA)}

On $27^{\text {th }}$ March 2001, the New Electricity Trading Arrangements (NETA) was introduced to replace the pool with the concept that markets participants have rights to transact electricity power by bilateral trading [5]. On $1^{\text {st }}$ April 2005, with the joining of Scottish network, the New Electricity Trading Arrangements (NETA) has been extended as the British Electricity Trading and Transmission Arrangements (BETTA) [5]. In the BETTA, electricity participants are able to trade by bilateral contracts. The bulk of electricity power is traded by Forward/Future contracts and short-term power exchange [8]. The Forward/Future contracts could be signed ahead of days, months or even years before actual power delivery [1]. The short-term power exchange is within 24 hours before electricity delivery which offers market participants an opportunity to adjust their contract details. Market participants are required to notify their predicted physical information in each half hour period, for seller is the planned generation output and for buyer is the metered demand, by 11 a.m. one day ahead. This information is called initial physical notifications (IPNs) [5]. Then market participants will hand in their final physical notifications (FPNs) to the SO before the gate closure, usually 3.5 hours before delivery [5]. Figure 2 show the structure of BETTA [5] and figure 3 shows the diagrammatic representation of BETTA time frame process [2]:

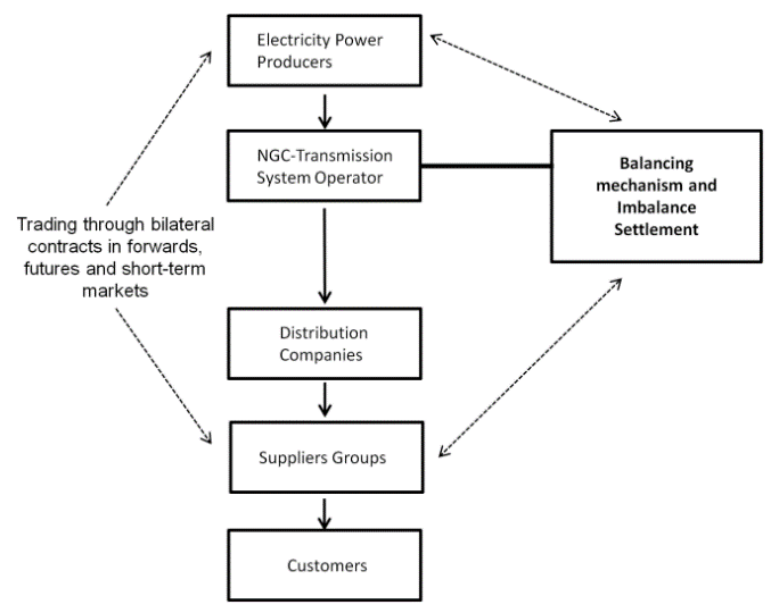

Fig. 2. The Structure of the BETTA

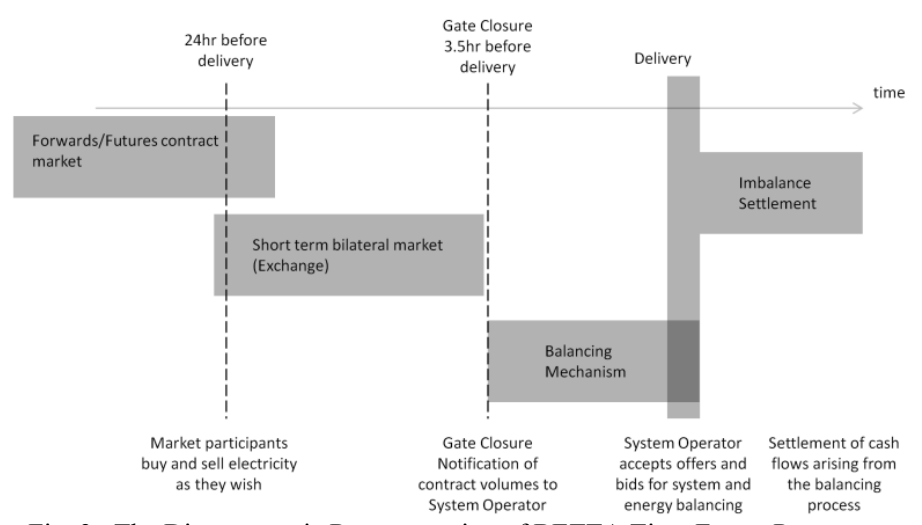

Fig. 3. The Diagrammatic Representation of BETTA Time Frame Process

Apart from physical data, participants have an opportunity to submit their offers and bids to the SO to take part in balancing mechanism which is on a voluntary basis [8]. The SO collects offers and bids then matches the balance between supply and demand so that system security is able to be ensured. After offers and bids have been accepted by the SO, market participants will be paid or charged according to their contracted prices. The SO will punish the participant who violates the contract.

Because more price information is available, bilateral market provides more liquidity so that electricity price decreases remarkable [8]. However, in order to avoid penalty from the SO, generators would increase output whilst suppliers would reduce demand which will make inefficient elements arise. Another problem is that renewable generation is unable to guarantee the production accurately so it will be easier penalized than other generations [8]. 


\section{B. Nordic Countries}

The Nordic electricity market is consisted of four markets from four countries: Sweden, Norway, Finland and Demark. In 1996, Sweden and Norway joined together and established the Norwegian-Swedish Exchange called as Nord pool which is the initial state of the current Nord pool [9]. Four years later, with Finland and Demark joining, a new integrated regulatory framework for electricity industry competition across Nordic countries was established which was also the first multinational electricity market.

The Nord Pool is consisted of two physical markets and several financial markets [10]. Figure 4 shows Nordic market's components [10]; Figure 5 shows the diagrammatic representation of Nord pool time frame process [2]:

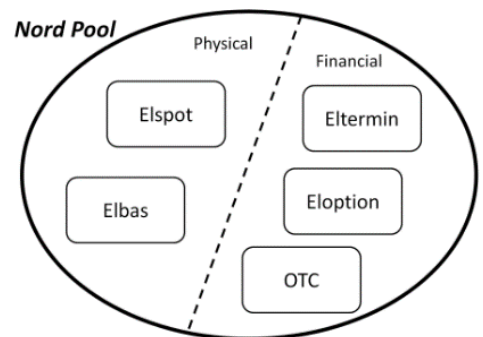

Fig. 4. Nordic Market Components

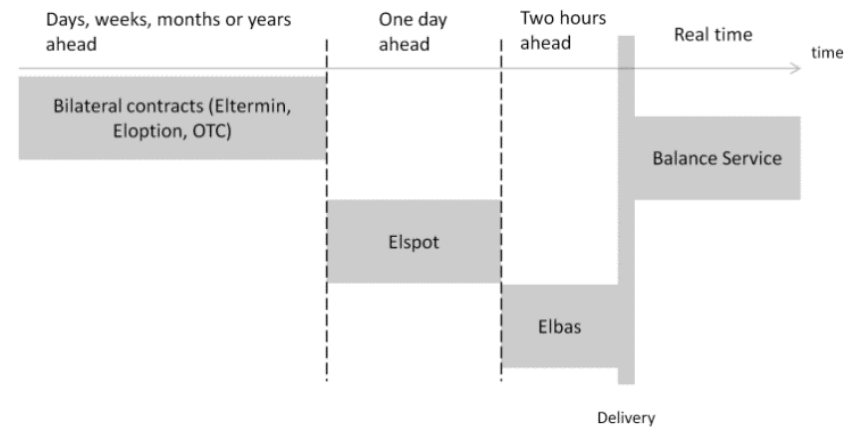

Fig. 5. The Diagrammatic Representation of Nord Pool Time Frame Process

Elspot market is referred as the main physical market which is a day-ahead market arranging hourly bilateral contracts. It will determine the hourly spot price which will be regarded as the reference price both for financial and physical market. About one thirds of electricity consumption was traded in Elspot market and the rest of transactions were taken place through bilateral contacts [10]. Elbas was seemed as a supplement of Elspot market for additional balancing services. It offers participants an opportunity to adjust their physical power position such as the price and volume of supply or demand within two hours period. There are two financial markets in Nord Pool including Eltermin and Eloption. Sellers and buyers can sign a forward and future contract in Eltermin market days, weeks, months and even up to four years ahead to hedge against the prices volatility risk. Eloption market is quite a new financial market which also provides contract services. The Nord Pool also allows brokers to organise the over the counter (OTC) trading for market participants to make bilateral contracts [2]. The Nord Pool power exchange is the key point of Nordic electricity market.
System price is determined in spot market [11]. When transmission network capacity is sufficient, the wholesale electricity prices in four countries are the same. Once transmission capacity shortage takes place, prices in different areas will be different. Norway will divide into several price areas; Sweden and Demark have two areas respectively; while Finland only has one area [10].

Sufficient hydropower and low demand make Nordic market a nearly wonderful power system [11]. Four electricity markets definitely dilute the market power [11] but due to congestion there are still chances for participants to exercise tricky games [2].

\section{PJM Interconnection, US}

PJM Interconnection is a regional transmission organization (RTO) in the United States [12]. It is serving the area of Pennsylvania, New Jersey, Maryland and other states in the eastern part of United States [13]. PJM is the biggest electricity wholesale market in the world which serves 61 million of customers and has 900 participant power companies. PJM has an energy market which is consisted of a day-ahead market and a real-time balancing market; a capacity credit market; a financial transmission right (FTR) auction market and an ancillary service market which is consisted of a regulation market and a pin reserve market [12]. Figure 6 shows PJM market components [12]:

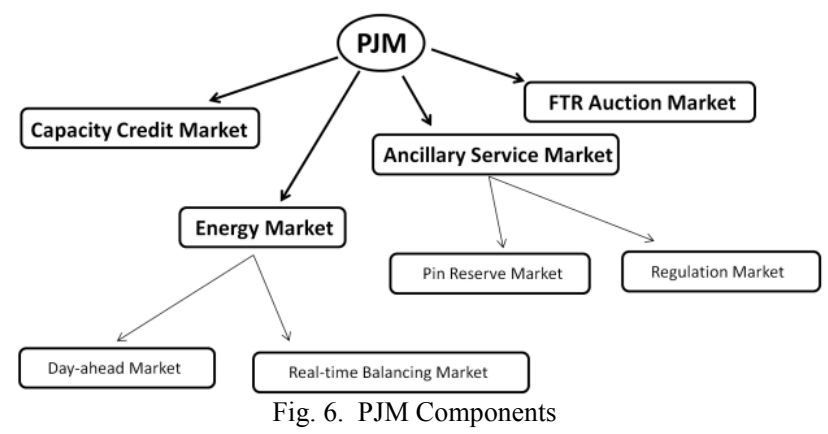

The day-ahead market calculates the hourly clearing prices for each hour of the next operating day based on generators' offers, demand bids, virtual supply offers, virtual demand bids and bilateral transaction schedules and those data will be submitted into the day-ahead market voluntarily [13]. The balancing market calculates the clearing price in every 5 minutes period with the principle of actual system operation and security constrained economic dispatch. Different settlement methods are adopted in two markets [13]. The dayahead market settlement follows the planned hourly power quantities and day-ahead hourly electricity prices. The balancing market settlement follows the hourly quantity deviations between planned values and real-time values. Both prices calculation are based on the mechanism of Locational Marginal Pricing (LMPs). The day-ahead market allows participants to exchange electricity at binding day-head prices. Participants can schedule bilateral transactions at binding day-ahead congestion price which is the price difference between source and sink. In the day-ahead market, generators who joined an installed capacity contract should 
hand in their offer schedules even if they are self-scheduled and during outage. Participants should submit bilateral transaction schedules to the SO to notify whether they are willing to pay congestion charges and be curtailed if transmission congestion occurs. As the day-ahead market closing, the SO starts to make the schedule and dispatch for each hour of the next operating day.

The real-time energy market is based on the real-time operation which is described by state estimator with the data from generation offers and transactions [13]. The generation rebidding period from 4 p.m. to 6 p.m. is introduced for the generators who are not selected into day-ahead market schedules to alter their bids. Generators who produce more than scheduled quantities will be paid by real-time LMPs. For customers who consume the power volume more than the planned quantities will be charged by real-time LMPs. If participants who exchange spot energy, their trades will be settled at real-time LMPs. Besides the day-ahead market and the real-time balancing market, PJM also provides financial instruments for participants to hedge the price variation risks.

PJM can provide market liquidity and price transparency. Efficient and sufficient price signals help market participants to make correct judgement.

\section{Generic Congestion Management Schemes}

\section{A. Uniform Marginal Price}

Uniform Marginal Price was implemented in the former England \& Wales Pool. It indicates electricity prices in the market will be identical for all participants without considering the locations [2]. The SO adopts the principle referred as "re-dispatch first, compensate later" to manage transmission congestion [6]. In dispatch stage, system operator collects all the generators' bids and ranks them from cheapest to higher prices until demand has been satisfied. The highest price becomes the market clearing price which is also called the system marginal price and the most expensive generator becomes the marginal generator. When transmission congestion occurred, the SO will re-dispatch the generators list, at the meantime ensuring the re-dispatch cost is the minimum. The algorithm of dispatch is shown as [6]:

Subject to:

$$
\min \sum_{i=1}^{N_{G}} C_{G_{i}}\left(P_{G_{i}}\right)
$$

$$
\begin{gathered}
\sum_{i=1}^{N_{G}} P_{G_{i}}=\sum_{j=1}^{N_{L}} P_{L_{j}} \\
0 \leq P_{G_{i}} \leq P_{G_{i}}^{\max }
\end{gathered}
$$

Where:

$$
\begin{aligned}
& C_{G_{i}}\left(P_{G_{i}}\right) \text { is the bid-based generation is cost of generator } i \\
& P_{G_{i}} \text { is the generator i power generation } \\
& P_{L_{j}} \text { is the demand prediction } \\
& N_{G} \text { is the total number of generators } \\
& N_{L} \text { is the total number of loads } \\
& P_{G_{i}}^{\max } \text { is the generator i maximum capacity }
\end{aligned}
$$

$[6]:$

If congestion occurs, an inequality constraint will be added

$$
P_{\text {line }_{k}} \leq P_{\text {line }_{k}}^{\max }
$$

Where:

$$
\begin{aligned}
& P_{\text {line }_{k}} \text { is the power flow on the line } k \\
& P_{\text {line }_{k}}^{\text {max }} \text { is the power flow up limitation of line } k
\end{aligned}
$$

Re-dispatched schedule is decided by the new algorithm. The chosen generators are called "constrained on" generators which were out-of-merit generators but is on the new generation list and those failed to be chosen ones are called "constrained off" generators but they were in-merit generators [2]. All on-list generators will be paid by PPP and also be compensated by an adjustment payment for the difference between their bid prices and the PPP. All customers will pay for PSP plus Uplift.

In this method, electricity trading price barely reflect the congestion cost. The SO allocates congestion cost equally on participants without considering power flow contribution. Uniform price is unable to provide investment incentives for long-term development.

\section{B. Locational Marginal Price}

Locational marginal price is the primary pricing scheme in the United States electricity markets for electricity price calculations and congestion management. Before calculate the LMP, the SO uses optimal power flow (OPF) to calculate dispatch of each generator [14]. OPF is the integration of power flow calculation and minimization of economic objective function subject to the equality and inequality constraints. As known, power injected into all nodes is equal to power withdrawn plus transmission losses which can be written as [2]:

Where:

$$
-\sum_{i=1}^{N} P_{i}^{g}+\sum_{i=1}^{N} P_{i}^{d}+P_{\text {loss }}=0
$$

$$
\begin{aligned}
& P_{\text {loss }} \text { is the transmission losses in the power system } \\
& \sum_{i=1}^{N} P_{i}^{g} \text { is the sum real power generated from node } i \\
& \sum_{i=1}^{N} P_{i}^{d} \text { is the sum real power demand at node } i
\end{aligned}
$$

Based on the equation (8), the the corresponding Lagrangian equation can be defined as follows [15]:

$$
\begin{aligned}
& \quad \mathcal{L}=\sum_{i=1}^{N} C_{i}\left(P_{i}^{g}\right)+\lambda_{0}\left(-\sum_{i=1}^{N} P_{i}^{g}+\sum_{i=1}^{N} P_{i}^{d}+P_{\text {loss }}\right)+ \\
& \sum_{l=1}^{L} \mu_{l}\left(\left|P_{l}\right|-P_{l}^{\max }\right)+\sum_{i=1}^{N} \pi_{i}^{\max }\left(P_{i}^{g}-P_{i, \max }^{g}\right)+ \\
& \sum_{i=1}^{N} \pi_{i}^{\min }\left(P_{i, \min }^{g}-P_{i}^{g}\right)
\end{aligned}
$$

Where:

$\lambda_{0}$ is the Lagrangian multiplier of the whole system's power balance constraint

$\mathrm{N}$ is the total number of nodes

$\mathrm{L}$ is the total number of transmission lines

$\mu_{l}$ is the Lagrangian multiplier of transmission line constraint $\pi_{i}^{\text {max }}$ and $\pi_{i}^{\text {min }}$ are the Lagrangian multipliers of maximum and minimum generation capacity of generator $i$

Based on the equation (9), equation of LMP of node $\mathrm{i}$ can be obtained as follow [2]: 
$L M P_{i}=\frac{\partial \mathcal{L}}{\partial P_{i}^{d}}=\lambda_{0}\left(1+\frac{\partial P_{l o s s}}{\partial P_{i}^{d}}\right)+\sum_{l=1}^{L} \mu_{l} \times \frac{\partial P_{l}}{\partial P_{i}^{d}}=\lambda_{0}+\lambda_{0} \times$ $\left(\frac{\partial P_{\text {loss }}}{\partial P_{i}^{d}}\right)+\sum_{l=1}^{L} \mu_{l} \times T_{i-1}$

(10)

Where: $l$ constraint

$T_{i-1}$ is the sensitivity factor for real power at node $i$ with line

LMP in node $i$ can be divided into three components as follows [16]:

(11)

$$
L M P_{i}=L M P_{i}^{\text {energy }}+L M P_{i}^{\text {congetion }}+L M P_{i}^{\text {loss }}
$$

Where:

$L M P_{i}^{\text {energy }}=\lambda_{0}$ is the system marginal cost of node $i$

$L M P_{i}^{\text {congestion }}=\lambda_{0} \times\left(\frac{\partial P_{\text {loss }}}{\partial P_{i}^{d}}\right)$ is the cost of transmission congestion of node $\mathrm{i}$ of node $i$

$L M P_{i}^{\text {loss }}=\sum_{l=1}^{L} \mu_{l} \times T_{i-1}$ is the cost of transmission losses

If there is no congestion and transmission loss, LMPs of each node will be the same which is set as the system marginal price. When congestion happens, LMPs between different nodes will become distinct due to transmission lost and congestion [14]. At one node, generator will be paid from LMPs and customer will pay for the LMPs and both actions will be implemented by the SO.

LMP system is transparent so that correct incentives and guidance for industry investment in particular zone can be provided. But pricing scheme is bid-based rather than costbased so that generators would still try to exercise market power. The SO can collect congestion revenue which will increase the possibility of market inefficiency [2].

\section{Zonal Price}

Zonal price simplifies the LMP which is a complex approach [17]. The SO splits the whole system into zones depending on historical data and economic operation of each node. Figure 7 shows the process [2]:

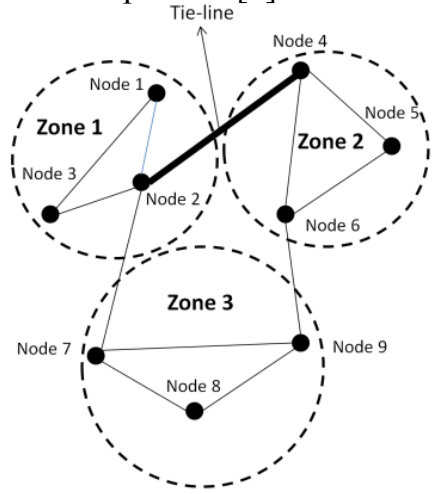

Fig. 7. Multi-nodes system split into three zones when congestion occurs

When there is no congestion, electricity prices in each node will be identical to the system marginal price which is calculated based on the bids collected from market participants. Once transmission congestion occurs, the whole system will be divided into different zones which were defined in advance. Inside a zone, electricity price is uniform which is calculated based on the bids from participants inside the zone.

From figure 7, node 1, 2, 3, node 4, 5, 6 and node 7, 8, 9 are respectively composed into three single zones. The transmission line between node 2 and node 4 now becomes the only connection which links zone 1 and zone 2 named "Tie-line" [2]. Zonal Price is actually the combination of the Uniform Marginal Price and the LMPs [18]. Inside each zone all nodal prices are identical which is similar with the Uniform Marginal Price. Each zone can be regarded as a "big" node which has its own electricity price priced by LMPs.

Zonal price may simplify the complex process of LMP while it is hard to define zone boundaries because it only considers the assumption that congestion inside zone is infrequent [2]. As a result, intra-zonal congestion problems are ignored easily by priced on average cost while it still exists [1]. Zonal price uses an invalid assumption to meet reality which brings chances for participants inside a zone to play games [18].

\section{Transaction Curtailments}

In bilateral market like BETTA, in order to ensure the security of power system operation, the SO will curtail transactions in order to reduce the power flow on the transmission branch. With different superposition models, transaction curtailment can be based on four rules [6]:

\section{- First Come First Serve:}

The SO will make generation schedule based on "first come first serve" principle. The last submitted transaction will be rigidly curtailed first without considering the power flow contribution on transmission network. If it is unable to relieve congestion, the second last submitted transaction will be the target and then one by one until congestion solved.

This method is rigid and didn't take power flow contribution into consideration [2]. Sometimes it is unable to relieve transmission congestion.

\section{- Pro Rata (Proportional):}

In this approach, the SO will curtail the power transaction volume proportionally based on the power flow contribution of each market participant. Market participant, who occupies transmission capacity more, will be curtailed more, vice versa [2].

Process of this method is fair and transparent.

- Minimum-net Curtailment:

This method is aim to restrict the curtailment volume to the minimum. Since different transaction curtailment has different power flow sensitivities to any line flow, curtail one certain transaction is much more effective than other transaction curtailment [6]. The SO is focus on searching such bilateral transaction to avoid waste curtailment. 
This process is transparent but not a market-based method so that it cannot provide incentives for industry investment [2]

\section{- $\quad$ Price Based}

\section{Compensative Price}

In bilateral market, no participant wants their contracts to be curtailed. If the curtailment is unavoidable, they want to receive compensation. In this method, participants are required to submit a compensation price at the money per MW that the SO pays to compensate curtailed transaction and will also be accepted by both generator and load [6]. When congestion occurs, the SO will curtail the lowest compensation price transaction in order to minimize the total cost of curtailment.

\section{Willingness to Pay}

In this method, markets participants are required to submit a price which describes how much they will pay for to avoid their transaction being curtailed. Of course, transaction from the participant who didn't submit the price or bid at the lowest price will be curtailed firstly by the SO [6].

This method doesn't consider the flow sensitivity and transmission contribution so it cannot be utilized alone [2].

\section{CONCLUSION}

In this article, four typical and successful deregulated electricity markets the old England \& Wales Pool, BETTA, Nordic Pool and PJM have been explained in details. Facts have proved that many countries around the world are working on liberalizing their electricity markets in order to achieve new requirements for power energy industry. However, with maturing competitive environment, issues which were easy to solved become complex, for example, transmission congestion. As a consequence, each market attempts to find approaches to relieve congestion based on their respective market rules and conditions. Hence, uniform marginal price in E\&W Pool, LMPs in PJM, zonal price in Nordic Pool and transaction curtailments in BETTA have been developed and adopted to manage congestion problems. But there is no perfect congestion management scheme and each method has its drawback. In bilateral market, transaction curtailments have different mechanisms and also different shortages such as causing extra cost for market participants, considering not comprehensive, providing no incentives and so on. In Pool and Hybrid market, LMP is obviously more effective than uniform price because it provides more economic information to participants and stronger incentives for long-term investments. Compared with Zonal price, LMP is still a wiser method for congestion relief because when it comes to market power LMP's performance seems to be more effective [19].

\section{REFERENCES}

[1] Kwok Lun Lo and Yee Shan Yuen, "Deregulation of Electric Utilities," in Power System Restructuring And Deregulation, book, Wiley, pp. 5074 ,

[2] J. Sun, "Demand Side Approaches for Congestion Management in Electricity Market," Ph.D. dissertation, Dept. EEE. Eng., Univ. Strathclyde, 2014.

[3] Kopsakangas-Savolainen, Maria, and Rauli Svento. "Real-Time Pricing; An Application to the Nordic Power Markets." In Modern Energy Markets, 29-44. Green Energy and Technology. Springer London, 2012

[4] R. D. Christie, B. F. Wollenberg, and I. Wangensteen, "Transmission management in the deregulated environment," Proceedings of the IEEE, vol. 88, pp. 170-195, 2000.

[5] G. Simmonds, "Regulation of the UK Electricity Industry," CRI Industry Brief, University of Bath, Desktop published by Jan Marchant 2002 Edition.

[6] M.P. Abdullah, "Congestion Management and Security Cost Allocation in Electricity Market," Ph.D. dissertation, Dept. EEE. Eng., Univ. Strathclyde, 2008.

[7] R. Dettmer, "Living with NETA [New Electricity Trading Arrangements]," IEE Review, vol. 48, pp. 32-36, 2002.

[8] S. Hesmondhalgh, "Is NETA the blueprint for wholesale electricity trading arrangements of the future?," Power Systems, IEEE Transactions on, vol. 18, pp. 548-554, 2003.

[9] O. Gjerde, "The deregulated Nordic electricity market-10 years of experience," presented at the Transmission and Distribution Conference and Exhibition 2002: Asia Pacific. IEEE/PES, 2002.

[10] L. Bergman, "The Nordic electricity market-continued success or emerging problems?," Swedish economic policy review, vol. 9, pp. 51$88,2002$.

[11] E. S. Amundsen and L. Bergman, "Why has the Nordic electricity market worked so well?," Utilities Policy, vol. 14, pp. 148-157, 2006.

[12] J. Tong, "Overview of PJM energy market design, operation and experience," in Electric Utility Deregulation, Restructuring and Power Technologies, 2004.(DRPT 2004). Proceedings of the 2004 IEEE International Conference on, 2004, pp. 24-27.

[13] A. L. Ott, "Experience with PJM market operation, system design, and implementation," Power Systems, IEEE Transactions on, vol. 18, pp. $528-534,2003$

[14] $\mathrm{H}$. Li and L. Tesfatsion, "ISO net surplus collection and allocation in wholesale power markets under LMP," Power Systems, IEEE Transactions on, vol. 26, pp. 627-641, 2011.

[15] T. Orfanogianni and G. Gross, "A general formulation for LMP evaluation," Power Systems, IEEE Transactions on, vol. 22, pp. 1163$1173,2007$.

[16] L. Fangxing and B. Rui, "DCOPF-Based LMP Simulation: Algorithm, Comparison with ACOPF, and Sensitivity," Power Systems, IEEE Transactions on, vol. 22, pp. 1475-1485, 2007.

[17] Holmberg, P., and E. Lazarczyk. "Congestion Management in Electricity Networks: Nodal, Zonal and Discriminatory Pricing." Cambridge Working Papers in Economics. Faculty of Economics, University of Cambridge, 2012.

[18] Z. Alaywan, T. Wu, and A. D. Papalexopoulos, "Transitioning the California market from a zonal to a nodal framework: an operational perspective," in Power Systems Conference and Exposition, 2004. IEEE PES, 2004, pp. 862-867.

[19] R. Green, "Nodal pricing of electricity: how much does it cost to get it wrong?," Journal of Regulatory Economics, vol. 31, pp. 125-149, 2007 